NIKHEF/2003-009

\title{
Supersymmetric hydrodynamics
}

\author{
T.S. Nyawelo ${ }^{\dagger}$ \\ NIKHEF, PO Box 41882 \\ 1009 DB Amsterdam \\ The Netherlands
}

\begin{abstract}
We work out some properties of a recently proposed globally $N=1$ supersymmetric extension of relativistic fluid mechanics in four-dimensional Minkowski space. We construct the lagrangean, discuss its symmetries and the corresponding conserved Noether charges. We reformulate the theory in hamiltonian formulation, and rederive the (supersymmetry and internal) transformations generated by these charges. Super-Poincaré algebra is also realized in this formulation.
\end{abstract}

† e-mail: tinosn@nikhef.nl 


\section{Introduction}

In recent years, there has been an increased interest in studying hydrodynamical systems from various points of view. The supersymmetric generalization of planar Chaplygin gas arising from supermembrane theory in $(3+1)$-dimensional spacetime was proposed in [1, 3. In $(1+1)$ dimensions, there is a large class of models. As an example we mention a supersymmetric fluid model which has been studied by Bergner and Jackiw [6]. Related developments in supersymmetrization of gas models can be found in [4, [5].

We have recently proposed a supersymmetric theory of hydrodynamics in fourdimensional Minkowski space, for irrotational flow, that is, when the vorticity -the circulation in the motion of fluid around a fixed point- is zero [7]. However, general fluid motions are not irrotational. It is therefore, desirable to explore the possibility of extending the model to a wider class of flows, such as flow with non-vanishing vorticity. However, in the presence of vorticity, the current Chern-Simons term could provide an obstruction to constructing a lagrangean for fluid motion. This obstruction is absent if the hydrodynamical current is decomposed into three scalars in so-called Clebsch ${ }^{1}$ parametrization 8 .

In a recent work [14 an alternative to the Clebsch decomposition of hydrodynamical currents in terms of complex potentials taking values in a Kähler manifold has been proposed. It introduces a general lagrangean density reproducing equations of motion of a perfect (dissipationless) relativistic fluid:

$$
\partial^{\mu} T_{\mu \nu}=0, \quad T_{\mu \nu}=p g_{\mu \nu}+(\varepsilon+p) u_{\mu} u_{\nu}, \quad \partial_{\mu} j^{\mu}=0, \quad j^{\mu}=\rho u^{\mu} .
$$

where $p$ is the pressure, $\varepsilon$ is the energy-density and $u^{\mu}$ is the velocity four-vector. The vanishing divergence of the fluid density current $j^{\mu}$ is the equation of continuity in hydrodynamics which expresses the conservation of the fluid density $\rho$ during the flow, whilst the divergence-free energy-momentum tensor $T_{\mu \nu}$ expresses the conservation of energy and momentum. In addition to (1), the Kähler parametrization allows a straightforward generalization to the case of superfields. Thus it can be used to construct a supersymmetric extension of relativistic fluid mechanics.

In this paper, we work out some properties of the supersymmetric extension of fluid mechanics proposed in 14 and discuss in details various aspects of this supersymmetric field theory. In section 2, we present the supersymmetric lagrangean in terms of superfields and work out its component form. In section 3 we discuss the internal symmetries of these lagrangeans in terms of Killing vectors, which represent infinitesimal symmetry transformations. Then we construct infinitesimal supersymmetry transformations of the fields appearing in the lagrangean. Using Noether's procedure, we construct the conserved quantities associated to these symmetries, such as supercharges, which are the generators of supersymmetry transformations, as well as the energy-momentum tensor from which the four-momentum is constructed. A canonical formulation of the theory in terms of a hamiltonian with a

\footnotetext{
${ }^{1}$ Parametrization of any vector field $A_{\mu}$ in terms of three scalar potential $(\alpha, \beta, \gamma)$ is called Clebsch parametrization: $A_{\mu}=\partial_{\mu} \alpha+\beta \partial_{\mu} \gamma$
} 
corresponding bracket structure is given in section 4. Section 5 contains the conclusions.

\section{Supersymmetric lagrangeans}

In this section, we construct a supersymmetric lagrangean, using the tensor calculus as described in [12. Our aim is to arrive at a recipe which will allow us to write down a general supersymmetric theory, so that later we can apply the results to the special case of a supersymmetric extension of relativistic fluid mechanics. As supersymmetric models in four dimensions require the target manifold of scalar fields to be a Kähler manifold, we summarize here the most important results concerning Kähler manifolds relevant for later discussions; for more detailed and complete discussion on Kähler manifolds see [9, 10, 11].

A Kähler manifold is a complex manifold, parametrized locally by $N$ complex coordinates $z^{\alpha}$ and their complex conjugates $\bar{z}^{\underline{\beta}}(\alpha, \beta=1, \ldots, N)$ on which a real line element can be defined by

$$
\mathrm{d} s^{2}=g_{\alpha \underline{\beta}} \mathrm{d} \bar{z} \underline{\beta} \mathrm{d} z^{\alpha},
$$

with a hermitian metric $g_{\alpha \underline{\beta}}$. The hermitian metric $g_{\alpha \underline{\beta}}$ is said to be Kählerian if the corresponding Kähler 2 -form $\omega=-i g_{\alpha \underline{\beta}} \mathrm{d} \bar{z}^{\underline{\beta}} \wedge \mathrm{d} z^{\alpha}$ is closed,

$$
\begin{aligned}
\mathrm{d} \omega= & -\frac{i}{2}\left(g_{\alpha \underline{\beta}, \gamma}-g_{\underline{\gamma}, \alpha}\right) \mathrm{d} z^{\gamma} \wedge \mathrm{d} z^{\alpha} \wedge \mathrm{d} \bar{z}^{\underline{\beta}} \\
& -\frac{i}{2}\left(g_{\alpha \underline{\beta}, \underline{\gamma}}-g_{\alpha \underline{\gamma}, \underline{\beta}}\right) \mathrm{d} z^{\alpha} \wedge \mathrm{d} \bar{z}^{\underline{\beta}} \wedge \mathrm{d} \bar{z}^{\underline{\gamma}}=0
\end{aligned}
$$

The comma denotes a derivative with respect to $z^{\alpha}$ and $\bar{z}^{\underline{\beta}}$. The requirement (3) is equivalent to the equations

$$
g_{\alpha \underline{\beta}, \gamma}=g_{\gamma \underline{\beta}, \alpha}, \quad g_{\alpha \underline{\beta}, \underline{\gamma}}=g_{\alpha \underline{\gamma}, \underline{\beta}} .
$$

From now on, we consider Kähler manifolds of complex dimension one, which are relevant for the following discussion. Locally, the metric can be derived from a scalar potential $K(z, \bar{z})$, the Kähler potential, as a second mixed derivate with respect to $z$ and $\bar{z}$

$$
g_{z \bar{z}}=K_{, z \bar{z}}
$$

The complex connections $\Gamma_{z z}^{z}$ and curvature tensor $R_{z \bar{z} z \bar{z}}$ of a Kähler manifold are given by

$$
\Gamma_{z z}^{z}=g^{z \bar{z}} g_{\bar{z} z, z}, \quad R_{z \bar{z} z \bar{z}}=g_{z \bar{z}, z \bar{z}}-g_{\bar{z} z, \bar{z}} g^{z \bar{z}} g_{\bar{z} z, \bar{z}}
$$

where $g^{z \bar{z}}$ is the inverse of the metric $g_{z \bar{z}}$. 
Having briefly discussed the Kähler geometry, we now turn to the construction of the action. It is defined in terms of two sets of chiral ${ }^{2}$ superfields $\Phi=\left(z, \eta_{+}, H\right)$, $\Lambda=\left(s, \chi_{+} . h\right)$ and a real vector multiplet $V=\left(C, \psi_{ \pm}, \mathcal{Z}, V_{\mu}, \lambda_{ \pm}, D\right)$. In terms of these multiplets, we propose the following action [14]

$$
S=\int d^{4} x \mathcal{L}, \quad \text { with } \quad \mathcal{L}=[V(K(\Phi, \bar{\Phi})+\Lambda+\bar{\Lambda})-\mathcal{F}(V)]_{D}
$$

Here $\mathcal{F}(V)$ is an analytic function of the real vector multiplet $V$. The component form of the action (17) after eliminating the auxiliary fields $D, \mathcal{H}, h, H, \chi_{+}, \lambda_{+}$and their complex conjugates reads

$$
\begin{aligned}
\mathcal{L}= & V^{\mu}\left(2 \partial_{\mu} N-i K_{, z} \partial_{\mu} z+i K_{, \bar{z}} \partial_{\mu} \bar{z}+2 i g_{z \bar{z}} \bar{\eta}_{-} \gamma_{\mu} \eta_{+}+\frac{i}{2} \mathcal{F}^{\prime \prime \prime}(C) \bar{\psi}_{+} \gamma_{\mu} \psi_{-}\right) \\
& -2 C\left(g_{z \bar{z}} \partial_{\mu} z \partial^{\mu} \bar{z}+g_{z \bar{z}} \bar{\eta}_{+} \stackrel{\leftrightarrow}{D} \eta_{-}-R_{z \bar{z} z \bar{z}} \bar{\eta}_{+} \eta_{+} \bar{\eta}_{-} \eta_{-}\right) \\
& -\frac{1}{2} \mathcal{F}^{\prime \prime}(C)\left[\partial_{\mu} C \partial^{\mu} C-V_{\mu} V^{\mu}+\bar{\psi}_{+} \stackrel{\leftrightarrow}{\partial} \psi_{-}\right]-\frac{2}{C} g_{z \bar{z}} \bar{\psi}_{+} \eta_{+} \bar{\psi}_{-} \eta_{-} \\
& +2 i g_{z \bar{z}}\left(\bar{\psi}_{+} \not \partial z \eta_{-}-\bar{\psi}_{-} \not \partial \bar{z} \eta_{+}\right)-\frac{1}{8} \mathcal{F}^{\prime \prime \prime \prime}(C) \bar{\psi}_{+} \psi_{+} \bar{\psi}_{-} \psi_{-} .
\end{aligned}
$$

In this expression we used the notation of the metric $g_{z \bar{z}}$, connection $\Gamma_{z z}^{z}$ and curvature $R_{z \bar{z} z \bar{z}}$, given in (5) and (6) respectively. The primes are the derivatives of $\mathcal{F}(C)$ w.r.t. its argument and $N=\operatorname{Im} s$ is the imaginary ${ }^{3}$ component of $s$. The Kähler covariant derivative of a chiral spinor and the left-right arrow above the covariant derivative are are defined by

$$
\begin{aligned}
& \not D \eta_{-}=\not \partial \eta_{-}+\bar{\Gamma}_{\bar{z} \bar{z}}^{\bar{z}} \not \partial \bar{z} \eta_{-}, \quad \bar{\psi}_{ \pm} \stackrel{\leftrightarrow}{\partial} \psi_{ \pm}=\bar{\psi}_{ \pm} \gamma^{\mu} \partial_{\mu} \psi_{ \pm}-\partial_{\mu} \bar{\psi}_{ \pm} \gamma^{\mu} \psi_{ \pm} \\
& \not D \eta_{+}=\not \partial \eta_{+}+\Gamma_{z z}^{z} \not \partial \eta_{+} .
\end{aligned}
$$

\section{$3 \quad$ Symmetries and currents}

In the this section, we discuss the symmetries of the theory described by the action (17), and the resulting conserved quantities. We first discuss internal symmetry, then we give the infinitesimal supersymmetry transformations. After that we construct the energy-momentum tensor following from the invariance of the action under translation.

Internal symmetries are realized as a set of holomorphic Killing vectors $R^{i}(z)$ and $\bar{R}^{i}(\bar{z})$, which are a solutions of the Killing equation

$$
R_{\bar{z}, z}^{i}+\bar{R}_{z, \bar{z}}^{i}=0
$$

\footnotetext{
${ }^{2}$ Our conventions for chiral spinors are given in the appendix

${ }^{3}$ We take this opportunity to point out that $N$ is the $\operatorname{Im} s$ and not $\operatorname{Re} s$ as it appeared in [7].
} 
Here $R_{\bar{z}}^{i}=g_{z \bar{z}} R^{i z}$. The Killing vectors generate the isometries of the manifold corresponding to the coordinate transformations

$$
\delta z=z^{\prime}-z=\Theta^{i} \delta_{i} z=\Theta^{i} R_{i}^{z}(z),
$$

with $\Theta^{i}$ the parameters of the infinitesimal transformations. The isometries define a Lie algebra of the isometry group $\mathcal{G}$, with structure constants $f_{i j}{ }^{k}$ via the Lie derivative by:

$$
\left(\mathcal{L}_{R_{i}}\left[R_{j}\right]\right)^{z}=R_{i}^{z} R_{j, z}^{z}-R_{j}^{z} R_{i, z}^{z}=f_{i j}{ }^{k} R_{k}^{z} .
$$

The infinitesimal transformations of other superfield components are found by requiring the isometries to commute with supersymmetry [13]

$$
\delta \eta_{+}=\Theta^{i} R_{i, z}^{z}(z) \eta_{+}, \quad \delta H=\Theta^{i}\left(R_{i, z}^{z}(z) H-R_{i, z z}^{z}(z) \bar{\eta}_{+} \eta_{+}\right) .
$$

If $\mathcal{G}$ has a subgroup $\mathcal{H}$ under which the transformations (11) are linear in $z$, we may visualize the manifold as a coset space $\mathcal{G} / \mathcal{H}$. A particularly simple example is provided by the coset space $S U(2) / U(1)$ with the Kähler potential

$$
K(\bar{z}, z)=\ln (1+\bar{z} z) .
$$

The explicit form of the Killing vectors (11) in the $S U(2) / U(1)$ case are

$$
\delta z=\epsilon+i \theta z+\bar{\epsilon} z^{2}=R^{z}(z)
$$

with $\Theta=(\theta, \epsilon, \bar{\epsilon})$. Here $\theta$ is the parameter of $U(1) \subset S U(2)$ phase transformation, and $(\bar{\epsilon}, \epsilon)$ are the complex parameters of the broken off-diagonal $S U(2)$ transformations. Under the isometry transformations (15) the Kähler potential $K(z, \bar{z})$ is invariant up to the real part of a holomorphic function $F(z)$ transforming in the adjoint representation of the algebra (12):

$$
\delta K(\bar{z}, z)=F(z)+\bar{F}(\bar{z}), \quad F(z ; \theta, \bar{\epsilon})=\frac{i}{2} \theta+\bar{\epsilon} z .
$$

The transformations of chiral superfield $\Lambda$ and the real vector superfield $V$ are obtained by requiring that the full action to be invariant under the isometry transformations (15):

$$
\delta \Lambda=-F(\Phi), \quad \delta V=0
$$

Observe, that the transformations of the scalar $N$ can therefore be written as

$$
\delta N=\frac{i}{2}(F(z)-\bar{F}(\bar{z}))
$$

The isometries (15) can be obtained locally as gradients of a set of real Killing potentials, defined by

$$
G(\theta, \epsilon, \bar{\epsilon})=i\left(K_{, z} R(z)-F(z)\right)=\frac{1}{2} \frac{\theta(1-\bar{z} z)+2 i(\epsilon \bar{z}-\bar{\epsilon} z)}{1+\bar{z} z} .
$$


Indeed, the variations (111) are given by

$$
\delta z=-i g^{z \bar{z}} G_{, \bar{z}}(\theta, \epsilon, \bar{\epsilon}) .
$$

Let us now return to the case of general $K(z, \bar{z})$ and construct the charges. A set of conserved currents can be derived using the Noether procedure from the isometry transformations (11), (13) and (18). The resulting currents are:

$$
\begin{aligned}
J_{\mu}(G)= & -2 V_{\mu} G-2 G_{; z}\left(i C \partial_{\mu} z-\bar{\psi}_{-} \gamma_{\mu} \eta_{+}\right)+2 G_{; \bar{z}}\left(i C \partial_{\mu} \bar{z}+\bar{\psi}_{+} \gamma_{\mu} \eta_{-}\right) \\
& +4 i C G_{; \bar{z} ; z} \bar{\eta}_{-} \gamma_{\mu} \eta_{+},
\end{aligned}
$$

where the semicolon denotes a covariant derivative using the connection (66). These currents are divergence free

$$
\partial \cdot J=0
$$

as it can be verified explicitly using the equations of motion. The field equations derived from lagrangean (8) read

$$
\begin{aligned}
& \mathcal{F}^{\prime \prime}(C) \square C=2 g_{z \bar{z}}\left(\partial z \cdot \partial \bar{z}+\bar{\eta}_{+} \stackrel{\leftrightarrow}{D} \eta_{-}\right)-\frac{1}{2} \mathcal{F}^{\prime \prime \prime}(C)\left[V^{2}+(\partial C)^{2}\right. \\
& \left.-\bar{\psi}_{+} \stackrel{\leftrightarrow}{\partial} \psi_{-}\right]-\frac{i}{2} \mathcal{F}^{\prime \prime \prime \prime}(C) \bar{\psi}_{+} Y \psi_{-}+\frac{1}{8} \mathcal{F}^{\prime \prime \prime \prime \prime}(C) \bar{\psi}_{+} \psi_{+} \bar{\psi}_{-} \psi_{-} \\
& -\frac{2}{C^{2}} g_{z \bar{z}} \bar{\psi}_{+} \eta_{+} \bar{\psi}_{-} \eta_{-}+2 R_{z \bar{z} z \bar{z}} \bar{\eta}_{+} \eta_{+} \bar{\eta}_{-} \eta_{-} \\
& \mathcal{F}^{\prime \prime}(C) V_{\mu}+2 \partial_{\mu} N=i\left(K_{, z} \partial_{\mu} z-K_{,, z} \partial_{\mu} \bar{z}-2 g_{z \bar{z}} \bar{\eta}_{-} \gamma_{\mu} \eta_{+}\right)-\frac{i}{2} \mathcal{F}^{\prime \prime \prime}(C) \bar{\psi}_{+} \gamma_{\mu} \psi_{-} \\
& \partial \cdot V=0, \\
& -2 C g_{z \bar{z}} \square z=2 i g_{z \bar{z}} \bar{\psi}_{-} \not \partial \eta_{+}+2 i g_{z \bar{z}, z} \bar{\psi}_{-} \not \partial z \eta_{+}-2 i g_{z \bar{z}}\left(V \cdot \partial z+\bar{\eta}_{+} \not \partial \psi_{-}\right) \\
& +g_{z \bar{z}, \bar{z}}\left(2 i \bar{\eta}_{-} Y \eta_{+}-\frac{2}{C} \bar{\psi}_{+} \eta_{+} \bar{\psi}_{-} \eta_{-}+2 i \bar{\psi}_{+} \not \partial z \eta_{-}\right. \\
& \left.-4 C \bar{\eta}_{-} \not \partial \eta_{+}+2 \bar{\eta}_{+} \not C \eta_{-}\right)+2 C g_{z \bar{z}} \partial C \cdot \partial z+2 C g_{z \bar{z}, z} \partial z \cdot \partial z \\
& +2 C R_{z \bar{z} z \bar{z}, \bar{z}} \bar{\eta}_{+} \eta_{+} \bar{\eta}_{-} \eta_{-}+4 C g_{\bar{z} z, \bar{z} z} \bar{\eta}_{+} \not \partial z \eta_{-}
\end{aligned}
$$

for the bosonic fields, and

$$
\begin{aligned}
\mathcal{F}^{\prime \prime}(C) \not \partial \psi_{+}= & -\frac{1}{2} \mathcal{F}^{\prime \prime \prime}(C)(\not \partial C+i Y) \psi_{+}-\frac{1}{4} \mathcal{F}^{\prime \prime \prime \prime}(C) \psi_{-} \bar{\psi}_{+} \psi_{+} \\
& -2 g_{z \bar{z}}\left(\frac{1}{C} \eta_{-} \bar{\psi}_{+}+i \not \partial \bar{z}\right) \eta_{+}, \\
4 C \not D \eta_{+}= & -2\left(\not \partial C-i \bigvee+\frac{1}{C} \psi_{-} \bar{\psi}_{+}\right) \eta_{+}-2 i \not \partial z \psi_{+} \\
& +4 C g^{z \bar{z}} R_{z \bar{z} z \bar{z}} \eta_{-} \bar{\eta}_{+} \eta_{+},
\end{aligned}
$$


for the fermionic ones. All other equations of motion for $\left(\bar{z}, \psi_{-}, \eta_{-}\right)$are obtained by complex conjugation of (26), (27) and (28) respectively. Expression (26) simplify considerably if we use the field equation (28) for $\eta_{+}$

$$
\begin{aligned}
-2 \mathcal{D} \cdot(C \partial z)= & 2 i \bar{\psi}_{-} \not D \eta_{+}-2 i\left(V \cdot \partial z+\bar{\eta}_{+} \not \partial \psi_{-}\right) \\
& +2 C g^{z \bar{z}} R_{z \bar{z} z \bar{z} ; \bar{z}} \bar{\eta}_{+} \eta_{+} \bar{\eta}_{-} \eta_{-}+4 C g^{\bar{z} z} R_{\bar{z} z \bar{z} z} \bar{\eta}_{+} \not \partial z \eta_{-} .
\end{aligned}
$$

Here the curly $\mathcal{D}$ represents a Kähler-covariant derivative:

$$
\mathcal{D}_{\mu}\left(\partial^{\mu} z\right)=\square z+\partial z \Gamma_{z z}^{z} \cdot \partial z
$$

The conserved charges $q(G)$ are obtained from the divergence free currents (22)

$$
q(G)=\int d^{3} x J^{0}(G)
$$

We now turn to the construction of supercharges. The infinitesimal supersymmetry transformations leaving the action (8) invariant, with anti-commuting chiral spinor parameters $\epsilon_{+}$and $\epsilon_{-}$are

$$
\begin{array}{ll}
\delta C=\frac{i}{2} \bar{\epsilon}_{+} \psi_{+}-\frac{i}{2} \bar{\epsilon}_{-} \psi_{-}, & \delta z=\bar{\epsilon}_{+} \eta_{+}, \\
\delta \psi_{+}=-\frac{1}{2}(Y+i \not \partial C) \epsilon_{-} & \delta \psi_{-}=-\frac{1}{2}(Y-i \not \partial C) \epsilon_{+} \\
\delta V_{\mu}=\bar{\epsilon}_{+} \sigma_{\mu \nu} \partial^{\nu} \psi_{+}+\bar{\epsilon}_{-} \sigma_{\mu \nu} \partial^{\nu} \psi_{-} & \delta \bar{z}=\bar{\epsilon}_{-} \eta_{-} \\
\delta N=\frac{1}{4} \mathcal{F}^{\prime \prime}(C)\left(\bar{\epsilon}_{+} \psi_{+}+\bar{\epsilon}_{-} \psi_{-}\right)+\frac{i}{2}\left(\bar{\epsilon}_{+} K_{, z} \eta_{+}-\bar{\epsilon}_{-} K_{, \bar{z}} \eta_{-}\right), & \\
\delta \eta_{+}=\frac{1}{2}\left(\not \partial z \epsilon_{-}+\epsilon_{+} \frac{1}{2 C} g^{z \bar{z}}\left(i \bar{\psi}_{+} \eta_{+}+2 C g_{z \bar{z}, z} \bar{\eta}_{+} \eta_{+}\right)\right) & \\
\delta \eta_{-}=\frac{1}{2}\left(\not \partial \bar{z} \epsilon_{+}+\epsilon_{-\frac{1}{2 C}} g^{z \bar{z}}\left(-i \bar{\psi}_{-} \eta_{-}+2 C g_{z \bar{z}, \bar{z}} \bar{\eta}_{-} \eta_{-}\right)\right) . &
\end{array}
$$

Under these transformations the variation of the lagrangean is a total derivative:

$$
\delta \mathcal{L}=\partial_{\mu}\left(\frac{i}{2} \bar{\epsilon}_{+} B_{+}^{\mu}-\frac{i}{2} \bar{\epsilon}_{-} B_{-}^{\mu}\right)
$$

with the vector-spinor $B_{ \pm}^{\mu}$ given, modulo equations of motion, by

$$
\begin{aligned}
B_{+}^{\mu} \simeq & 2 g_{z \bar{z}} \gamma^{\mu} \eta_{-} \bar{\psi}_{+} \eta_{+}+2 i C g_{z \bar{z}} \not \partial \bar{z} \gamma^{\mu} \eta_{+}-\frac{1}{2} \mathcal{F}^{\prime \prime}(C) \gamma^{\mu}(\not \partial C+i V) \psi_{+} \\
& -\frac{1}{2} \mathcal{F}^{\prime \prime \prime}(C) \gamma^{\mu} \psi_{-} \bar{\psi}_{+} \psi_{+} \\
B_{-}^{\mu} \simeq & 2 g_{z \bar{z}} \gamma^{\mu} \eta_{+} \bar{\psi}_{-} \eta_{-}-i 2 C g_{z \bar{z}} \not \partial z \gamma^{\mu} \eta_{-}-\frac{1}{2} \mathcal{F}^{\prime \prime}(C) \gamma^{\mu}(\not \partial C-i V) \psi_{-} \\
& -\frac{1}{2} \mathcal{F}^{\prime \prime \prime}(C) \gamma^{\mu} \psi_{+} \bar{\psi}_{-} \psi_{-} .
\end{aligned}
$$


where the similarity sign $\simeq$ in (34) signifies that the vector-spinors are given up to equations of motion. The supercurrents $S_{ \pm}^{\mu}$ following from the invariance of the action (7) under supersymmetry variations (32) are obtained using standard methods. By the usual Noether theorem, one finds for the supercurrent (and its hermitian conjugate) in terms of the variations of the complete set of fields $A$ :

$$
\frac{i}{2} \bar{\epsilon}_{+} S_{+}^{\mu}-\frac{i}{2} \bar{\epsilon}_{-} S_{-}^{\mu}=\frac{i}{2} \bar{\epsilon}_{+} B_{+}^{\mu}-\frac{i}{2} \bar{\epsilon}_{-} B_{-}^{\mu}-\frac{\delta \mathcal{L}}{\delta \partial_{\mu} A} \delta A,
$$

with $B_{ \pm}^{\mu}$ given in (34). A little work reveals that

$$
\begin{aligned}
S_{\mu+}= & 4 C g_{z \bar{z}} \gamma_{\mu} \eta_{-} \bar{\psi}_{+} \eta_{+}-4 i C g_{z \bar{z}} \not \partial \bar{z} \gamma_{\mu} \eta_{+}+\mathcal{F}^{\prime \prime}(C)(\not \partial C+i V) \gamma_{\mu} \psi_{+} \\
& -\frac{1}{2} \mathcal{F}^{\prime \prime \prime}(C) \gamma_{\mu} \psi_{-} \bar{\psi}_{+} \psi_{+}-2 i C g_{z \bar{z}, z} \gamma_{\mu} \eta_{-} \bar{\eta}_{+} \eta_{+}, \\
S_{\mu-}= & 4 g_{z \bar{z}} \gamma_{\mu} \eta_{+} \bar{\psi}_{-} \eta_{-}+4 i C g_{z \bar{z}} \not \partial z \gamma_{\mu} \eta_{-}+\mathcal{F}^{\prime \prime}(C)(\not \partial C-i V) \gamma_{\mu} \psi_{-} \\
& -\frac{1}{2} \mathcal{F}^{\prime \prime \prime}(C) \gamma_{\mu} \psi_{+} \bar{\psi}_{-} \psi_{-}+2 i C g_{\bar{z} z, \bar{z}} \gamma_{\mu} \eta_{+} \bar{\eta}_{-} \eta_{-} .
\end{aligned}
$$

The supercurrents and its hermitian conjugate are separately conserved:

$$
\partial_{\mu} S_{ \pm}^{\mu}=0
$$

as can be verified by use of equations of motion. From these currents one constructs the conserved spinors supercharges

$$
Q_{ \pm}=\int d^{3} x S_{ \pm}^{0}
$$

which are the generators of supersymmetry transformations.

Since supersymmetric field theories are translationally invariant, the theory described by lagrangean (8) conserves energy-momentum. By the Noether procedure, translation invariance leads to non-symmetric currents $\Theta_{\mu \nu}$ defined by

$$
\Theta_{\mu \nu}=-\frac{\delta \mathcal{L}}{\delta\left(\partial_{\mu} A_{i}\right)} \partial^{\nu} A_{i}+g_{\mu \nu} \mathcal{L}
$$

where $\mathcal{L}$ is the lagrangean (8), and the sum is taken over the various fields of the theory. The symmetrized version of the energy-momentum tensor $T_{\mu \nu}$ is obtained by the addition of an improvement term $\Omega_{\mu \nu}$ which is the anti-symmetric part of (39)

$$
\begin{aligned}
T_{\mu \nu}= & \mathcal{F}^{\prime \prime}(C)\left(\partial_{\mu} C \partial_{\nu} C+V_{\mu} V_{\nu}+\frac{1}{4} \bar{\psi}_{+} \gamma_{\{\mu} \stackrel{\leftrightarrow}{\partial}_{\nu\}} \psi_{-}\right)+\frac{i}{4} \mathcal{F}^{\prime \prime \prime}(C) \bar{\psi}_{+} V_{\{\mu} \gamma_{\nu\}} \psi_{-} \\
& +2 C g_{z \bar{z}}\left(\partial_{\mu} z \partial_{\nu} \bar{z}+\partial_{\mu} \bar{z} \partial_{\nu} z+\frac{1}{2} \bar{\eta}_{+} \gamma_{\{\mu} \stackrel{\leftrightarrow}{\partial}_{\nu\}} \eta_{-}\right)+i g_{z \bar{z}} \bar{\eta}_{-} V_{\{\mu} \gamma_{\nu\}} \eta_{+} \\
& -\left[i g_{z \bar{z}} \bar{\psi}_{+} \partial_{\{\nu} z \gamma_{\mu\}} \eta_{-}+\text {h.c. }\right]-\left[C g_{z \bar{z}, z} \bar{\eta}_{+} \partial_{\{\nu} z \gamma_{\mu\}} \eta_{-}+\text {h.c. }\right]+g_{\mu \nu} \mathcal{L},
\end{aligned}
$$


Here $\{\mu, \nu\}$ denotes the symmetrized expression. The conservation of the energymomentum tensor follows up on using the field equations. From this energy-momentum tensor one construct the conserved four-momentum

$$
P_{\mu}=\int d^{3} x T_{\mu 0} .
$$

In the following section, we construct the explicit expressions for the supercharges (38) and four-momentum vector (41).

\section{Canonical analysis}

In this section, we show that the supercharges $Q_{ \pm}$satisfy the supersymmetry algebra and that they generate the supersymmetry transformations (32) as well as the spacetime translations on the fields. As this action of the supersymmetry algebra in terms of $Q_{ \pm}$requires the use of canonical variables and hamiltonian equations of motion, we first present a canonical formulation of the theory and describe the dynamics in terms of phase-space coordinates and the hamiltonian. However in this formalism, the fermionic momenta turn out not to be independent degrees of freedom, as they are constrained to fermionic fields themselves. To eliminate these constraints we introduce Poisson-Dirac brackets, defined as the Poisson brackets from which the second class constraints have been projected out.

We now present details of this analysis. The canonical momenta of the theory are defined by

$$
\begin{aligned}
\pi_{C} & =\frac{\delta \mathcal{L}}{\delta \dot{C}}=\mathcal{F}^{\prime \prime}(C) \dot{C}, \quad \pi_{N}=\frac{\delta \mathcal{L}}{\delta \dot{N}}=2 V^{0}, \\
\pi_{z} & =\frac{\delta \mathcal{L}}{\delta \dot{z}}=i K_{, z} V_{0}+2 C g_{z z} \dot{\bar{z}}+2 i g_{z \bar{z}} \bar{\psi}_{+} \gamma^{0} \eta_{-}+2 C g_{z \bar{z}, z} \bar{\eta}_{+} \gamma^{0} \eta_{-}, \\
\bar{\pi}_{\bar{z}} & =\frac{\delta \mathcal{L}}{\delta \dot{\bar{z}}}=-i K_{\bar{z}} V_{0}+2 C g_{z \bar{z}} \dot{z}-2 i g_{z \bar{z}} \bar{\psi}_{-} \gamma^{0} \eta_{+}+2 C g_{z \bar{z}, \bar{z}} \bar{\eta}_{-} \gamma^{0} \eta_{+} \\
\pi_{\psi_{ \pm}} & =\gamma_{0} \frac{\delta \mathcal{L}}{\delta \dot{\bar{\psi}}_{\mp}}=\frac{1}{2} \mathcal{F}^{\prime \prime}(C) \psi_{ \pm}, \quad \pi_{\eta_{ \pm}}=\gamma_{0} \frac{\delta \mathcal{L}}{\delta \dot{\bar{\eta}}_{\mp}}=2 C g_{z \bar{z}} \eta_{ \pm} .
\end{aligned}
$$

Here we included $\gamma_{0}$ in the definition of the fermionic momenta so that the momenta of Majorana variables are Majorana themselves as well. Clearly, the last two equations of (42) are second-class constraints, expressing the fermionic momenta $\left(\pi_{\psi_{ \pm}}, \pi_{\eta_{ \pm}}\right)$in terms of fermionic fields:

$$
\chi_{\psi_{ \pm}}=\pi_{\psi_{ \pm}}-\frac{1}{2} \mathcal{F}^{\prime \prime}(C) \psi_{ \pm} \simeq 0, \quad \chi_{\eta_{ \pm}}=\pi_{\eta_{ \pm}}-2 C g_{z \bar{z}} \eta_{ \pm} \simeq 0
$$

The similarity sign $\simeq$ in last equality of (43) signifies that the constraints are defined

only on a subset (the physical shell) of the full phase space. In this extended phase 
space, the equal-time Poisson brackets of the theory are defined by

$$
\begin{aligned}
\left\{\pi_{\eta_{ \pm}}(\mathbf{r}), \bar{\eta}_{\mp}\left(\mathbf{r}^{\prime}\right)\right\} & =\left\{\pi_{\psi_{ \pm}}(\mathbf{r}), \bar{\zeta}_{\mp}\left(\mathbf{r}^{\prime}\right\}=\left\{\psi_{ \pm}\left(\mathbf{r}^{\prime}\right), \bar{\pi}_{\zeta_{\mp}}(\mathbf{r})\right\}=\gamma^{0} P_{\mp} \delta^{3}\left(\mathbf{r}-\mathbf{r}^{\prime}\right)\right. \\
\left\{\eta_{\mp}\left(\mathbf{r}^{\prime}\right), \bar{\pi}_{\eta_{ \pm}}(\mathbf{r})\right\} & =\gamma^{0} P_{ \pm} \delta^{3}\left(\mathbf{r}-\mathbf{r}^{\prime}\right), \quad\left\{N(\mathbf{r}), \pi_{N}\left(\mathbf{r}^{\prime}\right)\right\}=\delta^{3}\left(\mathbf{r}-\mathbf{r}^{\prime}\right), \\
\left\{z(\mathbf{r}), \pi_{z}\left(\mathbf{r}^{\prime}\right)\right\} & =\left\{\bar{z}(\mathbf{r}), \bar{\pi}_{\bar{z}}\left(\mathbf{r}^{\prime}\right)\right\}=\left\{C(\mathbf{r}), \pi_{C}\left(\mathbf{r}^{\prime}\right)\right\}=\delta^{3}\left(\mathbf{r}-\mathbf{r}^{\prime}\right),
\end{aligned}
$$

where $P_{ \pm}=\frac{1}{2}\left(1 \pm \gamma_{5}\right)$ are the left- and right-handed chiral projection operators.

In order to describe the canonical dynamics on the reduced phase-space determined by the constraint equations (43), we introduce Poisson-Dirac brackets

$$
\{A, B\}^{*}=\{A, B\}-\left\{A, \chi_{i}\right\} C_{i j}^{-1}\left\{\chi_{j}, B\right\}
$$

where $C_{i j}^{-1}$ is the inverse of the matrix of constraint brackets

$$
\begin{aligned}
C_{i j} & =\left\{\chi_{i}(\mathbf{r}, t), \bar{\chi}_{j}\left(\mathbf{r}^{\prime}, t\right)\right\} \\
& =-\left(\begin{array}{cccc}
0 & \mathcal{F}^{\prime \prime}(C) \gamma^{0} P_{-} & 0 & 0 \\
\mathcal{F}^{\prime \prime}(C) \gamma^{0} P_{+} & 0 & 0 & 0 \\
0 & 0 & 0 & 4 C g_{z \bar{z}} \gamma^{0} P_{-} \\
0 & 0 & 4 C g_{z \bar{z}} \gamma^{0} P_{+} & 0
\end{array}\right) \delta^{3}\left(\mathbf{r}-\mathbf{r}^{\prime}\right) .
\end{aligned}
$$

Applying this prescription, we obtain the full set of non-zero Poisson-Dirac brackets of our theory:

$$
\begin{aligned}
\left\{z(\mathbf{r}), \pi_{z}\left(\mathbf{r}^{\prime}\right)\right\}^{*} & =\left\{\bar{z}(\mathbf{r}), \bar{\pi}_{\bar{z}}\left(\mathbf{r}^{\prime}\right)\right\}^{*}=\left\{C,(\mathbf{r}) \pi_{C}\left(\mathbf{r}^{\prime}\right)\right\}^{*}=\delta^{3}\left(\mathbf{r}-\mathbf{r}^{\prime}\right) \\
\left\{\pi_{C}(\mathbf{r}), \bar{\psi}_{ \pm}\left(\mathbf{r}^{\prime}\right)\right\}^{*} & =\frac{\mathcal{F}^{\prime \prime \prime}(C)}{2 \mathcal{F}^{\prime \prime}(C)} \bar{\psi}_{ \pm} \delta^{3}\left(\mathbf{r}-\mathbf{r}^{\prime}\right), \quad\left\{\pi_{z}(\mathbf{r}), \bar{\eta}_{ \pm}\left(\mathbf{r}^{\prime}\right)\right\}^{*}=\frac{1}{2} \Gamma_{z z}^{z} \bar{\eta}_{ \pm} \delta^{3}\left(\mathbf{r}-\mathbf{r}^{\prime}\right) \\
\left\{\bar{\pi}_{\bar{z}}(\mathbf{r}), \bar{\eta}_{ \pm}\left(\mathbf{r}^{\prime}\right)\right\}^{*} & =\frac{1}{2} \Gamma_{\bar{z} \bar{z}}^{\bar{z}} \bar{\eta}_{ \pm} \delta^{3}\left(\mathbf{r}-\mathbf{r}^{\prime}\right), \quad\left\{\eta_{ \pm}(\mathbf{r}), \bar{\eta}_{\mp}\left(\mathbf{r}^{\prime}\right)\right\}^{*}=\frac{1}{4 C} g^{z \bar{z}} \gamma^{0} P_{\mp} \delta^{3}\left(\mathbf{r}-\mathbf{r}^{\prime}\right) \\
\left\{\psi_{ \pm}(\mathbf{r}), \bar{\psi}_{\mp}\left(\mathbf{r}^{\prime}\right)\right\}^{*} & =\frac{1}{2 \mathcal{F}^{\prime \prime}(C)} \gamma^{0} P_{\mp} \delta^{3}\left(\mathbf{r}-\mathbf{r}^{\prime}\right), \\
\left\{\eta_{ \pm}(\mathbf{r}), \pi_{z}\left(\mathbf{r}^{\prime}\right)\right\}^{*} & =-\frac{1}{2} \Gamma_{z z}^{z} \eta_{ \pm} \delta^{3}\left(\mathbf{r}-\mathbf{r}^{\prime}\right), \quad\left\{\eta_{ \pm}(\mathbf{r}), \bar{\pi}_{\bar{z}}\left(\mathbf{r}^{\prime}\right)\right\}^{*}=-\frac{1}{2} \Gamma_{\bar{z} \bar{z}}^{\bar{z}} \eta_{ \pm} \delta^{3}\left(\mathbf{r}-\mathbf{r}^{\prime}\right) \\
\left\{N(\mathbf{r}), \pi_{N}\left(\mathbf{r}^{\prime}\right)\right\}^{*} & =\delta^{3}\left(\mathbf{r}-\mathbf{r}^{\prime}\right), \quad\left\{\psi_{ \pm}(\mathbf{r}), \pi_{C}\left(\mathbf{r}^{\prime}\right)\right\}^{*}=-\frac{\mathcal{F}^{\prime \prime \prime}(C)}{2 \mathcal{F}^{\prime \prime}(C)} \psi_{ \pm} \delta^{3}\left(\mathbf{r}-\mathbf{r}^{\prime}\right)
\end{aligned}
$$

The Poisson-Dirac brackets of Noether charges (31) generate the isometries transformations, as defined in (11), (13) and (18)

$$
\delta_{M} A=\{q, A\}^{*},
$$

where the canonical Noether charges (31) is

$$
\begin{aligned}
q[G]= & \int d^{3} x\left[\pi_{N} G-g^{z \bar{z}} G_{z}\left(i \bar{\pi}_{\bar{z}}+\frac{1}{2} K_{\bar{z}} \pi_{N}\right)+g^{z \bar{z}} G_{\bar{z}}\left(i \pi_{z}-\frac{1}{2} K_{z} \pi_{N}\right)\right. \\
& \left.-2 i C\left(G_{z} \bar{\Gamma}_{\bar{z} \bar{z}}^{\bar{z}}+G_{\bar{z}} \Gamma_{z z}^{z}-2 G_{z \bar{z}}\right) \bar{\eta}_{-} \gamma_{0} \eta_{+}\right] .
\end{aligned}
$$


One may check the closure of the algebra of conserved charges by computing the Poisson-Dirac brackets of such two charges. After a long calculation, one finds that the result has the structure of a Poisson bracket on the 2-d manifold spanned by $(\bar{z}, z)$ :

$$
\left\{q\left[G^{(1)}\right], q\left[G^{(2)}\right]\right\}^{*}=q\left[G^{(3)}\right], \quad \text { with } \quad G^{(3)}=i g^{z \bar{z}}\left(G_{z}^{(1)} G_{\bar{z}}^{(2)}-G_{\bar{z}}^{(1)} G_{z}^{(2)}\right) .
$$

The canonical hamiltonian, obtained from lagrangean (8) by Legendre transformation, reads

$$
\begin{aligned}
& H=\int d^{3} x\left[\frac{1}{2 \mathcal{F}^{\prime \prime}(C)} \pi_{C}^{2}+\left(\frac{1}{8} \mathcal{F}^{\prime \prime}(C)+\frac{1}{8 C} g^{z \bar{z}} K_{, z} K_{, \bar{z}}\right) \pi_{N}^{2}+\frac{1}{2 C} g^{z \bar{z}} \pi_{z} \bar{\pi}_{\bar{z}}\right. \\
& -i \pi_{N}\left(\frac{1}{4} \mathcal{F}^{\prime \prime \prime}(C) \bar{\psi}_{+} \gamma_{0} \psi_{-}+g_{z \bar{z}} \bar{\eta}_{-} \gamma_{0} \eta_{+}\right)+\frac{1}{8} \mathcal{F}^{\prime \prime \prime \prime}(C) \bar{\psi}_{+} \psi_{+} \bar{\psi}_{-} \psi_{-} \\
& +\frac{1}{2} \mathcal{F}^{\prime \prime}(C)\left((\vec{\nabla} C)^{2}+\vec{V}^{2}++\bar{\psi}_{+} \stackrel{\leftrightarrow}{\nabla} \psi_{-}\right)+2 C g_{z \bar{z}}\left(\vec{\nabla} z \vec{\nabla} \bar{z}+\bar{\eta}_{+} \stackrel{\leftrightarrow}{\not} \eta_{-}\right) \\
& -2 C\left(R_{z \bar{z} z \bar{z}}-\Gamma_{z z}^{z} g_{z \bar{z}, \bar{z}}\right) \bar{\eta}_{+} \eta_{+} \bar{\eta}_{-} \eta_{-}+\frac{2}{C} g_{z \bar{z}} \bar{\psi}_{+} \gamma^{0} \eta_{-} \bar{\psi}_{-} \gamma^{0} \eta_{+}+ \\
& +\frac{2}{C} g_{z \bar{z}} \bar{\psi}_{+} \eta_{+} \bar{\psi}_{-} \eta_{-}+\left\{-\frac{i}{C} \bar{\psi}_{+} \gamma^{0} \eta_{-} \bar{\pi}_{\bar{z}}+\text { h.c }\right\}+i g_{z \bar{z}, \bar{z}} \bar{\eta}_{-} \eta_{-} \bar{\psi}_{+} \eta_{+} \\
& +\left\{\frac{i K_{, z}}{4 C g_{z \bar{z}}} \pi_{N} \bar{\pi}_{\bar{z}}+\text { h.c }\right\}-\left\{\frac{K_{, z}}{2 C} \pi_{N} \bar{\psi}_{-} \gamma^{0} \eta_{+}+\text {h.c }\right\}-i g_{z \bar{z}, z} \bar{\eta}_{+} \eta_{+} \bar{\psi}_{-} \eta_{-} \\
& \left.-\left\{2 i g_{z \bar{z}} \bar{\eta}_{-} \vec{\nabla} z \psi_{+}+2 C g_{z \bar{z}, z} \bar{\eta}_{+} \vec{\nabla} z \eta_{-}+\Gamma_{\bar{z} \bar{z}}^{\bar{z}} \pi_{z} \bar{\eta}_{-} \gamma^{0} \eta_{+}+\text {h.c }\right\}\right] .
\end{aligned}
$$

In this expression we have used for the 3-dimensional contraction $\not \nabla=\vec{\gamma} \cdot \vec{\nabla}$ a notation analogous to the 4-dimensional one. After a long and tedious calculation one finds that brackets with the hamiltonian reproduce all the field equations we derived earlier from the lagrangean (88):

$$
\partial_{0} A=\{A, H\}^{*} .
$$

We now turn to the construction of the canonical super-Poincaré algebra. First we construct the canonical expressions for the energy-momentum vector (41) and the supercharges $Q_{ \pm}$(38). For the four-momentum vector we find the result

$$
\begin{aligned}
P_{0}= & \int d^{3} x \mathcal{H}=H \\
P_{i}= & \int d^{3} x\left[-\pi_{C} \nabla_{i} C-\pi_{N} \nabla_{i} N--\frac{1}{2} \mathcal{F}^{\prime \prime}(C) \bar{\psi}_{+} \gamma_{0} \stackrel{\leftrightarrow}{\not} \psi_{-}-2 C g_{z \bar{z}} \bar{\eta}_{+} \gamma_{0} \stackrel{\leftrightarrow}{\not} \eta_{-}\right. \\
& -\left\{\frac{1}{8 C} K_{, \bar{z}} \pi_{N} \bar{\eta}_{-} \gamma_{i} \psi_{+}+\pi_{z}\left(\nabla_{i} z-\frac{i}{4 C} \bar{\eta}_{+} \gamma_{i} \psi_{-}\right)+\right. \\
& \left.\left.+\frac{i}{2} g_{z \bar{z}, z}\left(\bar{\eta}_{+} \gamma_{i} \psi_{-}\right)\left(\bar{\eta}_{+} \gamma_{0} \eta_{-}\right)+\text {h.c }\right\}\right] .
\end{aligned}
$$


It generates space time translations on the fields $A$ :

$$
\partial_{\mu} A=\left\{A, P_{\mu}\right\}^{*} .
$$

The phase-space supercharges $Q_{ \pm}$are obtained directly from the supercurrents (36), which reads explicitly

$$
\begin{aligned}
Q_{+}= & \int d^{3} \mathbf{r}\left[\left(\mathcal{F}^{\prime \prime}(C) \not \nabla C-2 i \not \nabla N+K_{, \bar{z}} \not \nabla \bar{z}-K_{, z} \not \nabla z\right) \gamma_{0} \psi_{+}\right. \\
& +\frac{1}{4} \mathcal{F}^{\prime \prime \prime \prime}(C) \gamma_{0} \psi_{-} \bar{\psi}_{+} \psi_{+}+\left(\pi_{C}-\frac{i}{2} \mathcal{F}^{\prime \prime}(C) \pi_{N}\right) \psi_{+}+\left(K_{, z} \pi_{N}-2 i \pi_{z}\right) \eta_{+} \\
& \left.-4 i C g_{z \bar{z}} \not \nabla \bar{z} \gamma_{0} \eta_{+}-g_{z \bar{z}} \gamma_{0} \eta_{-} \bar{\psi}_{+} \eta_{+}\right] \\
Q_{-}= & \int d^{3} \mathbf{r}\left[\left(\mathcal{F}^{\prime \prime}(C) \not \nabla C+2 i \not \nabla N+K_{, z} \not \nabla z-K_{, \bar{z}} \not \bar{z}\right) \gamma_{0} \psi_{-}+\right. \\
& +\frac{1}{4} \mathcal{F}^{\prime \prime \prime \prime}(C) \gamma_{0} \psi_{+} \bar{\psi}_{-} \psi_{-}+\left(\pi_{C}+\frac{i}{2} \mathcal{F}^{\prime \prime}(C) \pi_{N}\right) \psi_{-}+\left(K_{, \bar{z}} \pi_{N}+2 i \bar{\pi}_{\bar{z}}\right) \eta_{-} \\
& \left.+4 i C g_{z \bar{z}} \not \nabla z \gamma_{0} \eta_{-}-g_{z \bar{z}} \gamma_{0} \eta_{+} \bar{\psi}_{-} \eta_{-}\right] .
\end{aligned}
$$

It is now straightforwards to generate the infinitesimal supersymmetry transformation (32) by Poisson-Dirac bracketing with the spinor charges (55)

$$
\delta\left(\epsilon_{ \pm}\right) A= \pm \frac{i}{2}\left\{A, \bar{\epsilon}_{ \pm} Q_{ \pm}\right\}^{*} .
$$

These results show that the spinor charges $Q_{ \pm}$give the correct supersymmetry transformation of all the fields in the theory. The commutator of two such transformations with parameters $\epsilon^{1}, \epsilon^{2}$ indeed gives a translation with parameter $\xi^{\mu}=\frac{1}{2} \bar{\epsilon}_{+}^{2} \gamma^{\mu} \epsilon_{-}^{1}+$ h.c.:

$$
\left[\delta\left(\epsilon^{1}\right), \delta\left(\epsilon^{2}\right)\right] A=\xi^{\mu} \partial_{\mu} A .
$$

This can be verified explicitly by using the Poisson-Dirac brackets. Then equation (57) becomes:

$$
\pm \frac{i}{2}\left\{\left\{A, \bar{\epsilon}_{ \pm}^{2} Q_{ \pm}\right\}^{*}, \bar{\epsilon}_{ \pm}^{1} Q_{ \pm}\right\}^{*}-\left(\epsilon^{2} \leftrightarrow \epsilon^{1}\right)=\xi^{\mu} \partial_{\mu} A
$$

up to terms which vanish on-shell. By rearranging the terms in (58) using the Jacobi identity, we therefore have

$$
\pm \frac{i}{2}\left\{A,\left\{\bar{\epsilon}_{ \pm}^{2} Q_{ \pm}, \bar{\epsilon}_{ \pm}^{1} Q_{ \pm}\right\}^{*}\right\}^{*}=\xi^{\mu}\left\{A, P_{\mu}\right\}^{*},
$$

for any $A$. It follows that

$$
\pm \frac{i}{2}\left\{\bar{\epsilon}_{ \pm}^{2} Q_{ \pm}, \bar{\epsilon}_{ \pm}^{1} Q_{ \pm}\right\}^{*}=\xi^{\mu} P_{\mu}
$$


Now, by expanding out equation (60), one obtains the supersymmetry algebra relations

$$
\left\{Q_{ \pm}, \bar{Q}_{\mp}\right\}^{*}=2 P, \quad\left\{Q_{ \pm}, P_{\mu}\right\}^{*}=0 .
$$

The last equation of (61) follow immediately from (54). The brackets structure shows Poincare supersymmetry to be realized also in the canonical formulation of the theory.

\section{Summary and discussion}

In this paper we have presented a complete formulation of a particular supersymmetric field theory on an arbitrary Kähler manifold. We have started with the construction of the lagrangeans then, discussed various aspects of these general constructions. The supercurrents and the energy-momentum tensor following from the invariance of the action under supersymmetry and translation are constructed. Assuming that there exists an isometry group $G$ which leaves the lagrangean invariant, we have constructed the corresponding conserved isometry currents, in terms of Killing vector $R^{z}(z)$.

Next, we discussed in details the canonical formulation of the theory in terms of hamiltonian. We have explicitly constructed the canonical supercharges. We have shown that these supercharges generate the supersymmetry transformations, and satisfy the standard super-Poincaré algebra.

To get the hydrodynamical interpretation of the models, one has to relate the fields in our model to the particle number density $\rho$ and the velocity four-vector $u_{\mu}$, which is time-like $u_{\mu}^{2}=-1$. In particular, in the limit in which all fermion fields vanish, we have to identify the vector component $V_{\mu}$ with the particle number density $\rho$ and $u_{\mu}$ as in (11). This implies that $V_{\mu}$ is time-like four vector. However, this is not sufficient for this field theory to describe a relativistic model of hydrodynamics. We have to show that this identification is consistent with the field equation (25) which is the relativistic equation of continuity. Finally, to complete the hydrodynamical interpretation of the models describe by (8), it should be possible to write the bosonic part of the energy-momentum tensor (40) in standard form (11). The two energymomentum tensors (11) and (401) can be equivalent only in the hydrodynamical regime $\left(C, \eta_{ \pm} \rightarrow 0\right)$ of the model. This analysis has been discussed in [14.

\section{Acknowledgements}

I would like to thank J.W. van Holten and S. Groot Nibbelink for very useful and pleasant discussions during this work, which is part of the research programme Theoretical Subatomic Physics (FP52) of the Foundation for Fundamental Research of Matter (FOM) and the Netherlands Organization for Scientific Research (NWO). 


\section{A Appendix: Notations and conventions}

In this appendix we collect the conventions we used in this paper. Symmetrization of objects enclosed is denoted by braces $\{\ldots\}$, anti-symmetrization by the square brackets [...]; the total weight of such (anti-)symmetrization is always unit.

In this paper, we reformulated the theory in a hamiltonian formulation. To avoid a confusion, we have used the braces $\{$,$\} to denote the Poisson brackets, \{,\}^{*}$ to denote Poisson-Dirac brackets.

The Minkowski metric $g_{\mu \nu}$ has a signature $(-1,+1,+1,+1)$. Our conventions for chiral spinors

$$
\Sigma_{ \pm}=\frac{1 \pm \gamma_{5}}{2} \Sigma_{ \pm}, \quad \Sigma_{ \pm} \equiv\left(\lambda_{ \pm}, \psi_{ \pm}, \eta_{ \pm}, \chi_{ \pm}\right)
$$

are such, that $\gamma_{5} \Sigma_{ \pm}= \pm \Sigma_{ \pm}$and $\bar{\Sigma}_{ \pm} \gamma_{5}= \pm \bar{\Sigma}_{ \pm}$; charge conjugations acts as $\Sigma_{ \pm}=$ $C \bar{\Sigma}_{ \pm}^{T}$, where $\bar{\Sigma}_{ \pm}=i \Sigma_{\mp}^{\dagger} \gamma_{0}$. It should also be noted, that the euclidean $\gamma_{4}=i \gamma_{0}$ is hermitean, hence $\gamma_{0}$ is anti-hermitean. The charge-conjugation operator $C$ defined in the spinor space satisfies the properties:

$$
C=C^{\dagger}=C^{-1}=-C^{T}, \quad C \gamma_{\mu} C^{-1}=-\gamma_{\mu}^{T}
$$

where the superscript $T$ denotes transposition in spinor space. From this it follows, that

$$
C \gamma_{5} C^{-1}=\gamma_{5}^{T}, \quad C \sigma_{\mu \nu} C^{-1}=-\sigma_{\mu \nu}^{T} \quad \text { with } \quad \sigma_{\mu \nu}=\frac{1}{4}\left[\gamma_{\mu}, \gamma_{\nu}\right] .
$$

With these definitions one can show by taking the transposition of a scalar the following identities:

$$
\begin{aligned}
& \bar{\Sigma}_{ \pm} \mathbb{1 1} \Delta_{ \pm}=\bar{\Delta}_{ \pm} \mathbb{1} \Sigma_{ \pm}, \quad \bar{\Sigma}_{ \pm} \gamma_{\mu} \Delta_{ \pm}=-\bar{\Delta}_{ \pm} \gamma_{\mu} \Sigma_{ \pm}, \\
& \bar{\Sigma}_{ \pm} \gamma_{5} \Delta_{ \pm}=\bar{\Delta}_{ \pm} \gamma_{5} \Sigma_{ \pm}, \quad \bar{\Sigma}_{ \pm} \sigma_{\mu \nu} \Delta_{ \pm}=-\bar{\Delta}_{ \pm} \sigma_{\mu \nu} \Sigma_{ \pm} .
\end{aligned}
$$

Hermitian conjugation on bispinor reverses by definitions the order of the spinors $(\psi \chi)^{\dagger}=\chi^{\dagger} \psi^{\dagger}$ with no minus sign. Using this one can show that h.c. replaces $i \leftrightarrow-i$ and $+\leftrightarrow-$ :

$$
\left(\bar{\Sigma}_{ \pm} \Delta_{ \pm}\right)^{\dagger}=\bar{\Sigma}_{\mp} \Delta_{\mp}, \quad\left(\bar{\Sigma}_{ \pm} \not \partial \Delta_{\mp}\right)^{\dagger}=\bar{\Sigma}_{\mp} \not \partial \Delta_{ \pm}
$$

\section{References}

[1] R. Jackiw, and A. Polychronakos "Supersymmetric Fluid Mechanics" Phys. Rev. D62 (2000) 085019 hep-th/0004083.

[2] R. Jackiw, V.P. Nair, and So-Young Pi "Chern-Simons Reduction and nonAbelian Fluid Mechanics" Phys. Rev. D62 (2000) 085018 hep-th/0004084. 
[3] J. Hoppe "Supermembranes in 4 Dimensions" hep-th/9311059

[4] Mokhtar Hassaï "Supersymmetric Chaplygin gas" Phys. Lett. A290 (2001) 157-164; hep-th/0106252

[5] A. Das, and Z. Popowics "Supersymmetric Polytropic Gas Dynamics" hep-th/0109223

[6] Y. Bergner, R. Jackiw "Integrable Supersymmetric Fluid Mechanics from Superstrings" Phys. Lett. A284 (2001) 146-151; arXiv:physics/0103092.

[7] T.S. Nyawelo, S. Groot Nibbelink, and J.W. van Holten "Superhydrodynamics" Phys. Rev. D46 (2001) 02701; hep-th/0104104

[8] R. Jackiw "Lectures notes on Fluid Mechanics" arXiv:physics/0010042.

[9] B. Zumino "Supersymmetry and Kähler manifold" Phys. Lett. B87 (1979) 203.

[10] M. Nakahara "Geometry, topology and physics". Bristol, UK: Hilger (1995) 505 pages (Graduate student series in physics).

[11] J. R.O. Wells "Differential analysis on complex manifolds". New York, USA: Springer Verlag (1980) 260

[12] A. van Proeyen, "Superconformal tensor calculus in $\mathrm{N}=1$ and $\mathrm{N}=2$ Supergravity", proceedings of Karpacz Winter School, Karpacz, Poland, (1983)

[13] J.W. van Holten, Nucl. Phys. B260 (1985) 125

[14] T.S. Nyawelo, S. Groot Nibbelink, and J.W. van Holten "Relativistic hydrodynamics, Kähler manifolds and supersymmetry" hep-th/0307283 